\title{
Adaptive management and multi-objective optimization of virtual machine in cloud computing based on particle swarm optimization
}

\author{
Shuxiang $\mathrm{Li}^{1^{*}}$ and Xianbing Pan ${ }^{2}$
}

\author{
* Correspondence: \\ aichangfa19787@163.com \\ 'Department of Mathematics and \\ Physics Teaching, Yitong College, \\ Chongqing University of Posts and \\ Telecommunications, Hechuan \\ 401520, China \\ Full list of author information is \\ available at the end of the article
}

\begin{abstract}
In order to improve the adaptive management ability of virtual machine placement in cloud computing, an adaptive management and multi-objective optimization method for virtual machine placement in cloud computing is proposed based on particle swarm optimization (PSO). The objective optimization model of adaptive management of virtual machine placement in cloud computing is constructed by particle swarm evolution, and the global optimization control of adaptive management of virtual machine placement in cloud computing is carried out by introducing extremum perturbation operator. The global dynamic objective function of particle swarm optimization is constructed, and the global optimal solution of virtual machine in cloud computing is found by deconvolution algorithm, and the optimal position of particle swarm is searched in two-dimensional space. The multiobjective optimization problem of adaptive management of virtual machine placement is transformed into particle swarm optimization problem to realize adaptive management and multi-objective optimization of virtual machine placement in cloud computing. Simulation results show that the adaptive management of virtual machine placement in cloud computing using this method has better global optimization ability, better convergence of particle swarm optimization, and better performance of multi-objective optimization.
\end{abstract}

Keywords: Particle swarm optimization, Cloud computing, Virtual machine placement, Adaptive management, Multi-objective optimization

\section{Introduction}

Data centers usually use virtualization technology to provide all kinds of cloud storage or computing services for users. Virtualization technology can put multiple virtual machines on the same physical machine and can transparently migrate virtual machines from one physical machine to other physical machines, so the number of physical machines in the data center is greatly reduced and the hardware capital is improved. The source use rate reduces energy consumption. Efficient operation of the cloud data center requires flexible and convenient management of thousands of virtual machines.

(c) The Author(s). 2020 Open Access This article is licensed under a Creative Commons Attribution 4.0 International License, which permits use, sharing, adaptation, distribution and reproduction in any medium or format, as long as you give appropriate credit to the original author(s) and the source, provide a link to the Creative Commons licence, and indicate if changes were made. The images or other third party material in this article are included in the article's Creative Commons licence, unless indicated otherwise in a credit line to the material. If material is not included in the article's Creative Commons licence and your intended use is not permitted by statutory regulation or exceeds the permitted use, you will need to obtain permission directly from the copyright holder. To view a copy of this licence, visit http://creativecommons.org/licenses/by/4.0/. 
Virtual machine management includes two important steps, including virtual machine selection and placement, involving many indicators, such as resource use (CPU, memory, network IO, and magnetic disk IO), application performance, and power consumption $[1,2]$.

At present, most of the host selection methods only consider the use of resources. One of the three indexes of application performance and power consumption can only minimize the temperature or increase the utilization rate of resources or reduce power consumption. But it cannot be effective at the same time [3, 4]. However, an efficient cloud data center needs to reduce the power consumption at the same time as satisfying the user's application performance, which needs to consider the relationship among resource utilization, application performance, and power consumption [5, 6]. But it is not easy to consider these state indicators together because they conflict and constrain each other. For example, the integration of virtual machines into a small number of physical machines and the shutdown of idle physical machines can effectively reduce energy consumption, but the integration of virtual machines will lead to competition of physical resources, which will affect application performance and may cause server hotspots. To study host selection algorithm, it is necessary to consider temperature, resource usage, application performance, and power consumption, and optimize virtual machine placement, so that the data center can obtain lower temperature while effectively guaranteeing the application performance, higher resource utilization, and less power consumption $[7,8]$.

Many scholars at home and abroad have studied the problem of virtual machine placement. In [9], the problems of hardware resource management and application performance assurance in data center are studied. By analyzing the relationship between resource utilization and application performance, the relationship model between them is constructed. A virtual machine dynamic placement algorithm based on online migration technology is proposed. In [10], Dr. Ma pointed out that the rapidly increasing computational intensive application load in the current data center makes the temperature of the processor higher and the thermal energy distribution out of balance, thus increasing the power consumption of the cooling system and shortening the service life of the server. In order to effectively balance the processor temperature of each node in the data center, the idea of closing the hot node is proposed. In [11], the low complexity linear thermal cycle model is used to define the load allocation problem based on peak temperature in order to reduce the processor peak temperature to minimize the cooling cost. In [12], the node integration of cloud computing data center virtualization is described as a random packing optimization problem, but only processor resources are considered, and memory is not considered. In [13], an adaptive management framework for virtual machine placement in cloud computing is proposed, and a multi-objective genetic algorithm for virtual machine placement with application service-level target constraints is proposed, which can be used to formulate virtual machine placement strategies in the framework. However, the problem of energy consumption is not considered, and the resource control and energy consumption control are not combined, while the application load is concentrated, its performance is not guaranteed. In order to maximize the quality of service (QoS), the idle physical machine is used as the migration virtual machine to reposition the destination host, but this will increase the site of resources and energy consumption of the physical machine [14, 15]. 
Some achievements have been made in the field of virtual machine management, but there are some shortcomings $[16,17]$. Firstly, most of the research on placement strategy is based on single objective optimization under certain criteria. The optimal placement obtained by this method is only the optimal solution of certain conditions and cannot be compared with each other under various criteria. An effective placement strategy should consider multiple objectives and make trade-offs and compromises. Then, there are conflicts and constraints among the state factors, and no unified optimization model is established to deal with the optimization $[18,19]$.

In order to solve the above problems, this paper puts forward the adaptive management of virtual machine in cloud computing the particle swarm optimization algorithm in place and multi-objective optimization method based on multi-objective optimization model to construct adaptive management of virtual machine in cloud computing placed by particle swarm evolution method; the introduction of extreme disturbance operators are placed in a virtual machine in cloud computing global adaptive management optimization control, construction of global dynamic objective function of particle swarm optimization; using deconvolution method to find global optimal virtual machine in cloud computing is placed solution; the optimal position of particle swarm in 2-dimensional space search, adaptive management of virtual machine is placed in the multi-objective optimization problem and is transformed into particle swarm optimization. Finally, simulation experiments show this method in adaptive management to realize virtual machine placement in cloud computing and multiobjective optimization of superior performance.

\section{Methods}

\subsection{Objective optimization function of adaptive management}

Aiming at the multi-objective optimization of virtual machine placement technology, the particle swarm optimization (PSO) method is used to construct an adaptive management target optimization model of virtual machines in cloud computing [20, 21]. It is assumed that there are 8 migrating virtual machines and 5 physical machines. The particle population is adapted to obtain new individuals in the process of multiobjective optimization. The operator is:

$$
p_{\text {id }}^{\text {new }}=\left\{\begin{array}{lc}
p_{\text {id }}+m()\left(X_{\text {max }}-p_{\text {id }}\right) & \text { if } m()>0 \\
p_{\text {id }}+m()\left(p_{\text {id }}-X_{\text {min }}\right) & \text { if } m() \leq 0
\end{array}\right.
$$

In which, $X_{\max }$ and $X_{\min }$ are the maximum and minimum position value of particles. $m()$ is the wavelet function of each migration virtual machine, which is defined as:

$$
m()=\frac{1}{\sqrt{a}} e^{-\frac{\phi^{2}}{2 a^{2}}} \cos \frac{5 \phi}{a}
$$

In which, $\phi \in[-2.5 a, 2.5 a], a \in[1,10000]$. Given the migration virtual machine and the destination host selected for each iteration, the wavelet high frequency mutation can make the algorithm easier to search in the solution space.

Assumption 1: $f(D(x, \xi)) \leq f(x)$, and if the adaptive management crossover operator $\xi \in U$ is placed in cloud computing, then $f(D(x, \xi)) \leq f(x)$, where $D$ is a random optimization algorithm, $\xi$ is the solution found by adaptive management of virtual 
machine placement in cloud computing, $U$ is the feasible solution space, and $f$ is the objective function.

Assumption 2: For the adaptive management Borel subset $A$ of virtual machines placed in any cloud computing of $\mathrm{U}$, if its management measure $v(A)>0$, then $\prod_{k=0}^{\infty}(1-$ $\left.u_{k}(A)\right)=0$, where $u_{k}(A)$ is the adaptive optimization algorithm $D$ for placement of virtual machines in cloud computing, $D$ in the $k$ iteration, the probabilistic measure of the solution found on $U$.

Theorem 1: Suppose that the adaptive management objective function $\left\{x_{g, k}\right\}_{k=0}^{\infty}$ of virtual machine placement in cloud computing is a measurable function, and the solution space $\lim _{t \rightarrow \infty} P\left[x_{g, k} \in R_{\varepsilon}\right]=1$ is a measurable set, and the solution sequence generated by the algorithm is $P\left[x_{g, k} \in R_{\varepsilon}\right]$, then $x_{g, k} \in R_{\varepsilon}$ holds, where VMbest and PMbest represent the migration virtual machine and the destination host selected by each iteration, $k$ is the evolutionary algebra, and $x_{g, k} \in R_{\varepsilon}$ is the optimal solution found by the $k$ generation.

Record the ordered pair and update the current virtual machine migration scheme. The algorithm starts iterating through the steps described above until the optimal virtual machine migration scheme is obtained, and the standard PSO algorithm for adaptive management of virtual machine placement in cloud computing is described as:

$$
D\left(p_{g}(t), p_{i}(t)\right)= \begin{cases}p_{g}(t) & f\left(p_{g}(t)\right) \leq f\left(x_{i}(t)\right) \\ x_{i}(t) & f\left(p_{g}(t)\right)>f\left(x_{i}(t)\right)\end{cases}
$$

It is easy to prove that it satisfies the assumption 1 in the convergence criterion.

\subsection{Multi-objective optimization problem description}

The objective optimization model of adaptive management of virtual machine placement in cloud computing is constructed by particle swarm evolution method [22, 23]. The global optimization control of adaptive management of virtual machine placement in cloud computing is carried out by introducing extremum perturbation operator. The particle size of the swarm is $N$, and the sample space of the swarm must contain $U$, that is $U \subseteq \bigcup_{i=1}^{N} M_{i, k}$, where $M_{i, k}$ is the support set of the sample space of particle $\forall A \subset U$ in the $k$ generation. There are $N \times M$ combinations of ordered pairs between $N$ virtual machines and $v(A)>0$ physical computers. For BV, the measure placed by virtual machines in cloud computing, each one corresponds only to the running cost of virtual machine $V M i$ on the $P M j$ of physical machines $\operatorname{Cost}(V M i, P M j)$, and its speed is even higher. The new formula is convergent, and the improved ICLPSO algorithm converges to the global optimal solution provided it is proved that the interval algebra of particle cloning is finite.

All approximate solutions of intermediate particles can be regarded as a point in state space $S 2$. Let $s_{i}=\left(x_{1}, x_{2}, \ldots, x_{n}\right) \in S^{1}$ denote the first state in the state space $S 1$ in which the virtual machine is placed adaptively, and $f\left(s_{i}\right)=\left(f\left(x_{1}\right), f\left(x_{2}\right), \ldots, f\left(x_{n}\right)\right)$ denote that the random variable A processes the state $s^{*}=\{x \in X \mid f(x)=\max f(x)\}$ at the $k$ generation, so that the control objective of adaptive management of the virtual machine placement can be achieved. Function $f(x)$ is the fitness function on $X$, and $s^{*}=\{x \in X \mid f(x)=\max$ 
$f(x)\}$ is used to represent the optimal solution of the state space of adaptive management placed by the virtual machine.

Where $I=\left\{i \mid s_{i} \geq s_{j}, \forall s_{j} \in S^{1}\right\}$, an ordered pair corresponding to the minimum value is selected among all the resulting function values [24, 25], where VMbest and PMbest represent the migration virtual machine and destination host selected by the algorithm for each iteration, such as VMbest and PMbest satisfies:

$$
f\left(x_{1}\right)=f\left(x_{2}\right)=\ldots=f\left(x_{n}\right)=f^{*}
$$

The amount of state migration placed by virtual machines in cloud computing is $s_{i} \in$ $s^{*}$. Let the transition probability of stochastic process $\left\{A_{k}\right\}$ be:

$$
\begin{aligned}
& p_{i j}(k)=p\left\{A_{k+1}^{j} / A_{k}^{i}\right\} \geq 0, \text { where } \\
& p_{i j}(k)=p\left\{A_{k+1}^{j} / A_{k}^{i}\right\}=\sum_{s_{c} \in S^{2}} p\left\{C_{k}^{l} / A_{k}^{i}\right\} p\left\{C_{k+1}^{l} / A_{k}^{i} C_{k}^{l}\right\}
\end{aligned}
$$

In which, $i \in I, j \notin I$, for any physical machine migration variable $L$, there are:

$$
p\left\{A_{k+1}^{j} / A_{k}^{i} C_{k}^{l}\right\}=\sum_{s_{c} \in S^{2}} p\left\{C_{k}^{l} / A_{k}^{i} B_{k}^{b}\right\} p\left\{A_{k+1}^{j} / A_{k}^{i} B_{k}^{b} C_{k}^{l}\right\}
$$

The update of virtual machine migration scheme will cause a state change. It is necessary to recalculate the affected element values in cost matrix CostMatrix for multiobjective optimization control $[26,27]$.

\section{Multi-objective optimization for adaptive management of virtual machine placement}

The deconvolution algorithm is used to find the global optimal solution of virtual machine placement in cloud computing, searching the optimal position of particle swarm in two-dimensional space, according to the definition of clonal selection can be obtained:

$$
p\left\{A_{k+1}^{j} / A_{k}^{i} B_{k}^{b} C_{k}^{l}\right\}=0
$$

According to the minimum negative value in the cost difference matrix, the corresponding ordered pair is found, the ordered pair is stored, and the current optimal migration scheme is updated [28, 29]. The effective transfer function of the migration scheme is $p_{i j}(k)=0$. When $i \notin I, j \in I$, the local search process is satisfied.

$$
p_{i j}(k) \geq p\left\{C_{k}^{j} / A_{k}^{i}\right\} p\left\{A_{k+1}^{j} / A_{k}^{i} C_{k}^{j}\right\}
$$

Thus,

$$
\begin{aligned}
& p\left\{C_{k}^{j} / A_{k}^{i}\right\}=\sum_{s_{c} \in S^{2}} p\left\{B_{k}^{b} / A_{k}^{i}\right\} p\left\{C_{k+1}^{j} / A_{k}^{i} B_{k}^{b}\right\} \geq p\left\{B_{k}^{b} / A_{k}^{i}\right\} p\left\{C_{k}^{l} / A_{k}^{i} B_{k}^{j}\right\}=0 \\
& p\left\{A_{k+1}^{j} / A_{k}^{i} C_{k}^{l}\right\} \geq \sum p\left\{C_{k}^{j} / A_{k}^{i} B_{k}^{b}\right\} p\left\{A_{k+1}^{j} / A_{k}^{i} B_{k}^{b} C_{k}^{j}\right\} \geq p\left\{C_{k}^{j} / A_{k+1}^{j} A_{k}^{i} B_{k}^{j}\right\}=0
\end{aligned}
$$

Denote $p_{i}(k)=p_{i}\left(A_{k}^{i}\right), p_{k}=\sum_{i \notin I} p_{i}(k)$, during each iteration, the conditional probability that the current node is always replaced by the best neighbor node is: 


$$
p_{k+1}=\sum_{s_{c} \in S} \sum_{j \neq I} p_{i}(k) p_{i j}(k)=\sum_{i \in I} \sum_{j \neq I} p_{i}(k) p_{i j}(k)+\sum_{i \notin I} \sum_{j \notin I} p_{i}(k) p_{i j}(k)
$$

Because

$$
\begin{aligned}
& \sum_{i \notin I} \sum_{j \notin I} p_{i}(k) p_{i j}(k)+\sum_{i \notin I} \sum_{j \in I} p_{i}(k) p_{i j}(k)=\sum_{i \notin I} p_{i}(k)=p_{k} \sum_{i \notin I} \sum_{j \notin I} p_{i}(k) p_{i j}(k)=p_{k}- \\
& \sum_{i \notin I} \sum_{j \in I} p_{i}(k) p_{i j}(k) .
\end{aligned}
$$

Then,

$$
0 \leq p_{k+1} \leq p_{k}-\sum_{i \notin I} \sum_{j \in I} p_{i}(k) p_{i j}(k) \leq p_{k} \leq 1
$$

Using deconvolution algorithm to find the global optimal solution of virtual machine placement in cloud computing, there are:

$$
\lim _{k \rightarrow \infty} p_{k}=0
$$

The best migration scheme of virtual machine is obtained so that the sum of running cost of all migration virtual machines in the data center can be satisfied:

$$
1 \geq \lim _{k \rightarrow \infty} \sum_{s_{i} \cap \wedge^{*} \neq \phi} p_{i}(k) \geq \lim _{k \rightarrow \infty} \sum_{i \in I} p_{i}(k)=1-\lim _{k \rightarrow \infty} p_{k}=1
$$

In the formula, $\overline{\mathbf{C}}$ is the long-term covariance matrix of $M \times M$ dimension, and $\tilde{\mathbf{C}}$ represents the short-term covariance matrix of $M \times M$ dimension. Of which:

$$
\begin{aligned}
& \bar{C}_{i j}=\sum_{\tau}\left(x_{i \tau}-\bar{x}_{i \tau}\right)\left(x_{j \tau}-\bar{x}_{j \tau}\right) \\
& \tilde{C}_{i j}=\sum_{\tau}\left(x_{i \tau}-\tilde{x}_{i \tau}\right)\left(x_{j \tau}-\tilde{x}_{j \tau}\right)
\end{aligned}
$$

The global dynamic objective function of particle swarm optimization is constructed, and the global optimal solution of virtual machine placement in cloud computing is found by deconvolution algorithm; then, the multi-objective optimization problem can be reduced to the following optimization problem:

$$
\min J(\boldsymbol{\beta})=\log \frac{\boldsymbol{\beta} \overline{\mathbf{C}} \boldsymbol{\beta}^{T}}{\boldsymbol{\beta} \tilde{\mathbf{C}} \boldsymbol{\beta}^{T}}
$$

In order to increase the utilization rate of hardware resources as much as possible, the target host selection algorithm needs to select the most fully used physical computer as the destination host. CPU, memory, network IO, and disk IO are important hardware resources for physical computers. Using particle swarm optimization, the optimal population will not degenerate [30,31]. Based on the above analysis, adaptive management and multi-objective optimization of virtual machine placement in cloud computing based on particle swarm optimization are realized [31-33]. 


\section{Experiment}

In order to test the application performance of this method in multi-objective optimization of virtual machine placement, the simulation experiment is carried out. The design of Matlab 7 is sampled, and the threshold value is 0.9. The running cost of VMi on PMJ is 0.23 . The number of particles is 40 , the acceleration factor $c 1=c 2=$ 1.49445 , the mutation probability of virtual machine placement is 0.7 , and the maximum iterative algebra is 2000. Each algorithm runs 30 times independently. According to the above simulation parameters, the virtual machine placement adaptive management and multi-objective optimization are carried out. The sample set of virtual machine placement target optimization test is shown in Fig. 1.

Taking Fig. 1 as the test sample set, adaptive management and multi-objective optimization control are carried out, and the optimized output sample set is shown in Fig. 2.

Figure 2 shows that the adaptive management of virtual machine placement in this paper has better multi-objective optimization performance, and the convergence of different multi-objective optimization is tested. The result is shown in Fig. 3.

The analysis shows that based on the given optimization objective function and virtual machine cost operation model, the target host selection problem is modeled mathematically. Compared with the global search algorithm, the global search algorithm not only has a higher search efficiency than the global search algorithm, it can get a better migration scheme of virtual machine.

The experiment simulates 15 physical nodes and 39 virtual machines, and the dynamic resource requirement information of each application virtual machine is random. It is assumed that the resource requirements information of each application is known, including the forecast information of resource needs for each application in the future. In the initial distribution state of virtual machine, about $6 \%$ of physical nodes are overheated. At present, the resource requirements of virtual machines consider only twodimensional resources (processors and memory). The size of each generation in each

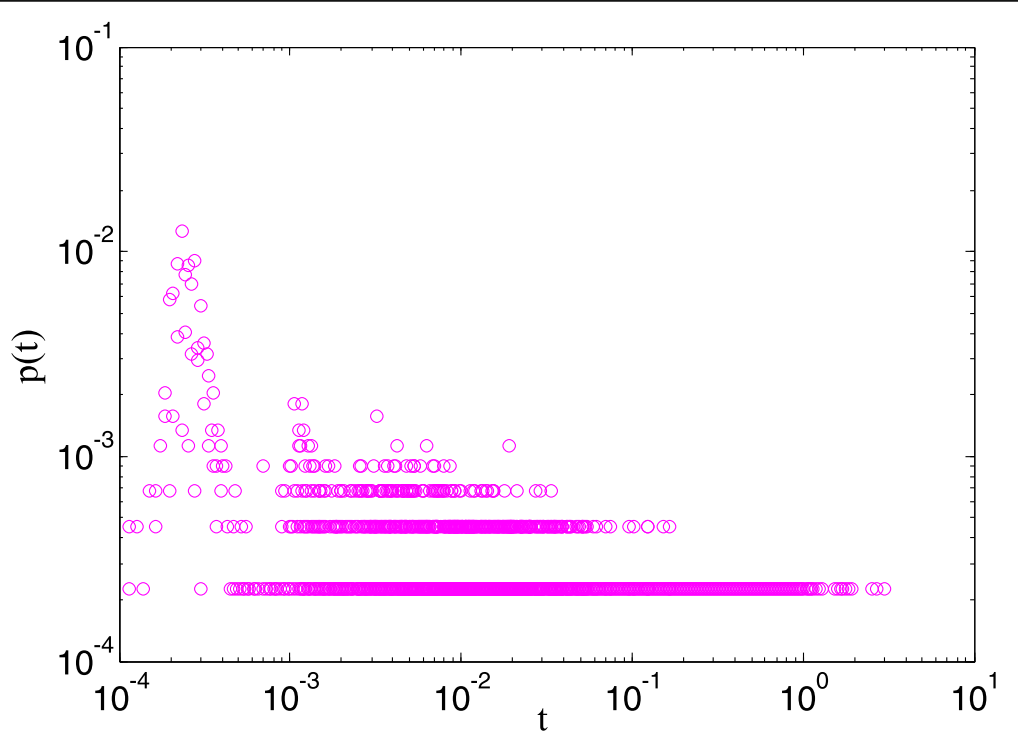

Fig. 1 Virtual machine placement target optimization test sample set 


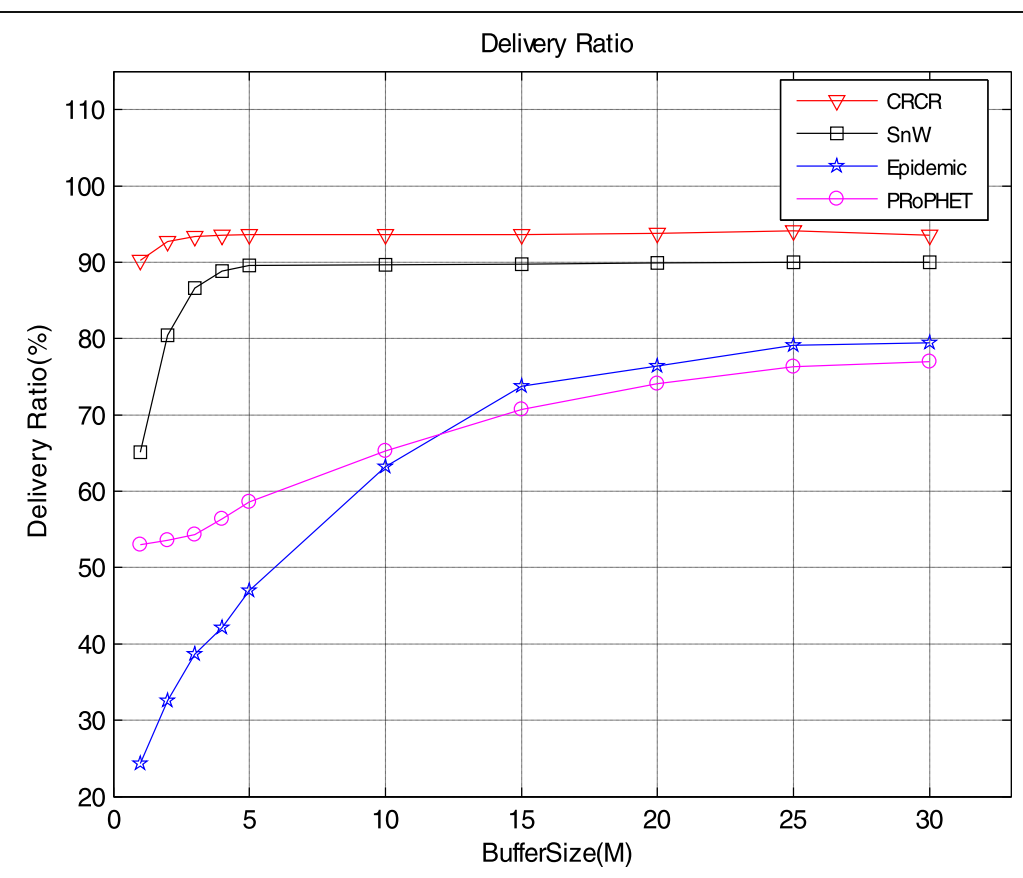

Fig. 2 Virtual machine placement target optimization training sample set

particle swarm optimization algorithm is set to 42 , and the total population is set to 36 . Under the same initial state of virtual machine distribution and the load change of the same application virtual machine, the new distribution state of each virtual machine is obtained by running this method and reference [6], reference [7], and reference [8]. The migration times, the stable time, and the number of physical nodes running of

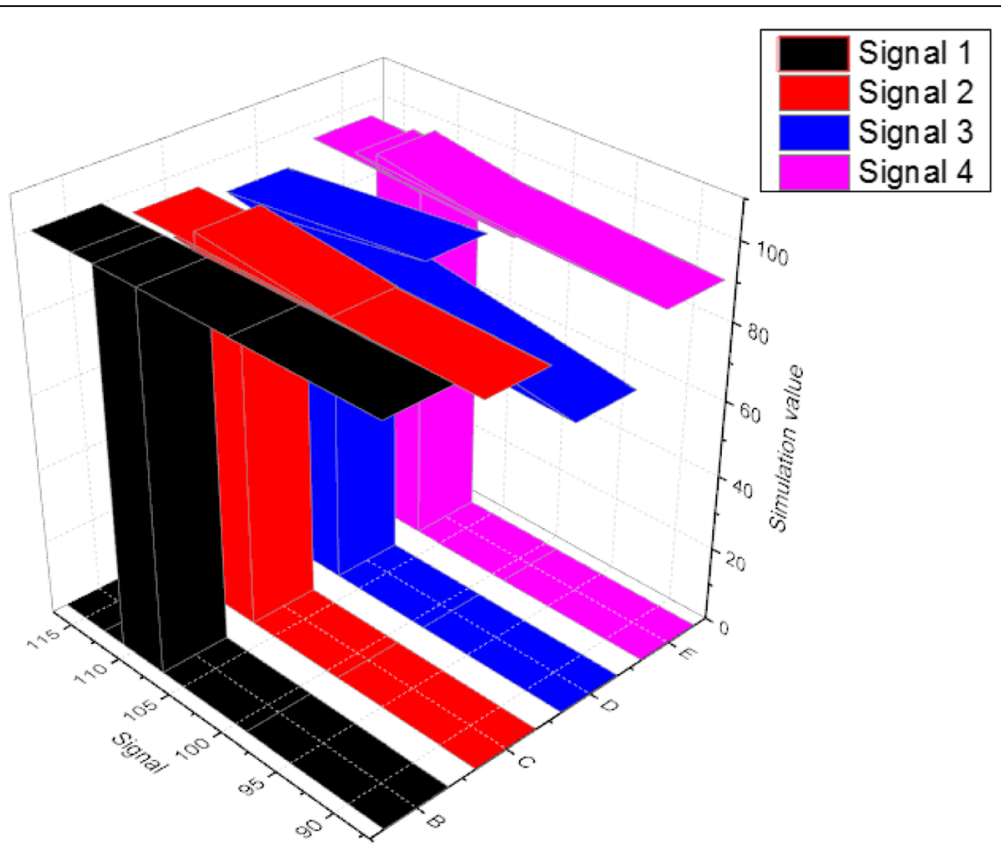

Fig. 3 Particle swarm evolution curve of our algorithm 
Table 1 Performance comparison results

\begin{tabular}{llll}
\hline Methods & Migration times & Stable time & Number of physical nodes running \\
\hline The proposed method & 50.3 & 11.3 & 3.2 \\
Reference [6] & 60.5 & 5.6 & 5.6 \\
Reference [7] & 70.3 & 3.2 & 7.3 \\
Reference [8] & 49.2 & 6.3 & 10.3 \\
\hline
\end{tabular}

these new distributed states are shown in Table 1. The results of the new distributed state run are shown in Fig. 4.

In Table 1, we can find that paper method makes a good balance between the stability of the virtual machine distribution and the virtual machine migration overhead required by the new and old states. It ensures that the new multi-virtual machine distribution state not only has a long stable time, but also changes from the current distribution state of the virtual machine to the virtual machine distribution state. The number of machine migration and the number of physical nodes are relatively small. Although reference [9] can only be transferred to the new virtual machine distribution state through the minimum number of virtual machine migration, but the distribution state of the new virtual machine can only be maintained for a relatively short time. Once the virtual machine distribution becomes unstable, it will stimulate new dynamic resource configuration requests and create a new virtual machine migration.

\section{Results and discussion}

In this paper, an adaptive management and multi-objective optimization method for virtual machine placement in cloud computing is proposed based on particle swarm optimization (PSO). The objective optimization model of adaptive management of virtual machine placement in cloud computing is constructed by particle swarm evolution, and the global optimization control of adaptive management of virtual machine placement in cloud computing is carried out by introducing extremum perturbation

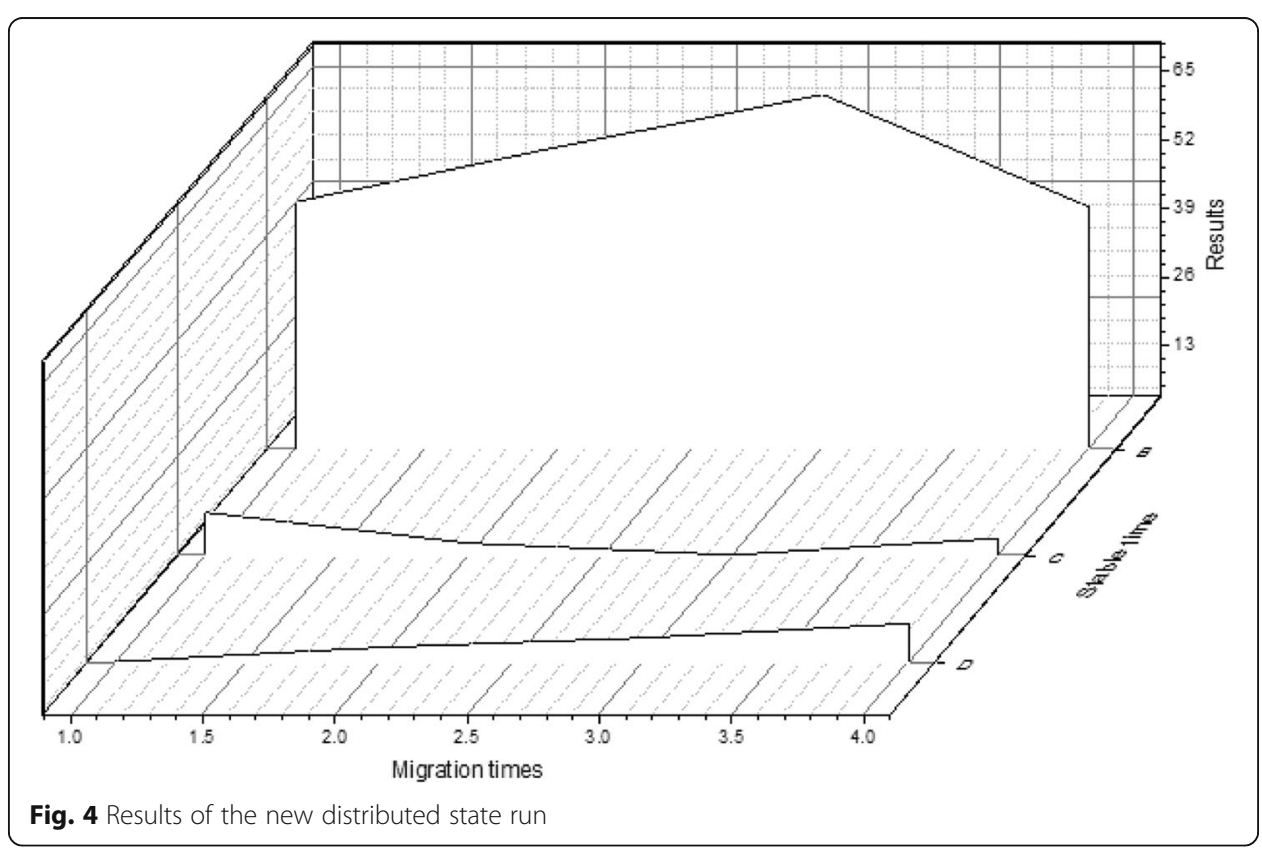


operator. The global dynamic objective function of particle swarm optimization is constructed, the global optimal solution of virtual machine in cloud computing is found by deconvolution algorithm, and the optimal position of particle swarm is searched in two-dimensional space. The multi-objective optimization problem of adaptive management of virtual machine placement is transformed into particle swarm optimization problem to realize adaptive management and multi-objective optimization of virtual machine placement in cloud computing. Simulation results show that the adaptive management of virtual machine placement in cloud computing using this method has better global optimization ability, better convergence of particle swarm optimization, and better performance of multi-objective optimization. This method has very good application value in the cloud computing virtual machine placement optimization management.

In this paper, the above work has been completed, and some research results have been obtained. However, due to the limitation of time and experimental environment, there are many solutions to be further optimized and perfected.

(1) The scheduling model is further improved. Integrating the resources in the cloud environment, optimizing the structure of the virtual resources of the data center, promoting the standardized management of the cloud computing resources, realizing the accurate and efficient and timely adaptive management of the cloud computing virtual machine layout, and improving the quality of the cloud computing service.

(2) We need to continue to improve the particle swarm optimization. On the basis of this algorithm, we optimize the rules of particle elimination, improve the accuracy of parameter adjustment, and improve the algorithm of premature processing.

(3) Although the simulation algorithm can get better experimental results, but the actual situation will be more complex, so the future work can build a formal experimental environment to verify the feasibility of the algorithm.

Abbreviation

CPU: Central processing unit

Acknowledgements

None

Informed consent

All authors agree to submit this version and claim that no part of this manuscript has been published or submitted elsewhere.

Authors' contributions

Shuxiang Li wrote the entire article. Xianbing Pang is responsible for data preprocessing. The author(s) read and approved the final manuscript.

Authors' information

Shuxiang Li, Department of Mathematics and Physics Teaching, Yitong College, Chongqing University of Posts and Telecommunications. Research direction: Combinatorial Mathematics and its application, Chongqing, Hechuan 401520 Xianbing Pan, Director, Department of Management Engineering, School of Communication, Chongqing University of Posts and Telecommunications. Main research direction: Applied Mathematics, Chongqing, Hechuan 401520.

Funding

None

Availability of data and materials

The datasets used and/or analyzed during the current study are available from the corresponding author on reasonable request. 
Ethics approval and consent to participate

This article does not contain any studies with human participants or animals performed by any of the authors.

\section{Competing interests}

The authors declare that they have no conflict of interest.

\section{Author details}

${ }^{1}$ Department of Mathematics and Physics Teaching, Yitong College, Chongqing University of Posts and Telecommunications, Hechuan 401520, China. ${ }^{2}$ Department of Management Engineering, School of Communication, Chongqing University of Posts and Telecommunications, Hechuan 401520, China.

Received: 8 January 2020 Accepted: 23 April 2020

Published online: 14 May 2020

\section{References}

1. H. Hongwei, G.E. Xiaotian, C. Xuansong, Density clustering method based on complex learning classification system. J. Comput. Appl. 37(11), 3207-3211 (2017)

2. C. Hao, C. Ping, H. Yang, et al., Empirical analysis of offshore and onshore RMB interest rate pricing: Based on the spillover index and its dynamic path. Int. Financ. Res. 350(6), 86-96 (2016)

3. Y. Lei, Y.W. Xin, Research on the policy effect of incremental expansion of margin and securities lending: Based on the multi period DID model and Hausman's test. Int. Financ. Res. 349(5), 85-96 (2016)

4. M. Bai, Y. Qin, Short-sales constraints and liquidity change: Cross-sectional evidence from the Hong Kong market. Pac. Basin Financ. J. 26, 98-122 (2014)

5. J. Yuying, J. Songbo, Study on the influence of the introduction of leverage ratio on the asset structure of commercial banks. Int. Financ. Res. 350(6), 52-60 (2016)

6. Y. Ding, H. Dai, S. Wang, Image quality assessment scheme with topographic independent components analysis for sparse feature extraction. Electron. Lett. 50(7), 509-510 (2014)

7. L.C. Manikandan, R.K. Selvakumar, A new survey on block matching algorithms in video coding. Int. J. Eng. Res. 3(2), $121-125$ (2014)

8. H. Bdi, L.J. Williams, Principal component analysis. Wiley Interdisciplinary Reviews: Computational Statistics 2(4), 433-459 (2010)

9. D. Shuangfeng, K. Lü, Z. Rui, D. Jiyang, Lung segmentation method based on 3D region growing method and improved convex hull algorithm. JEIT 38(9), 2358-2364 (2016)

10. M.A. Guodong, J. Jinbao, J. Xianzhen, An improved fletcher-reeves conjugate gradient method with descent property. Acta Mathematicae Applicatae Sinica 38(1), 89-97 (2015)

11. T. Zhongquan, The asymptotic relation between the maxima and sums of discrete and continuous time strongly dependent Gaussian processes. Acta Mathematicae Applicatae Sinica 38(1), 27-36 (2015)

12. A. Kumar, R. Pooja, G.K. Singh, Design and performance of closed form method for cosine modulated filter bank using different windows functions. International Journal of Speech Technology 17(4), 427-441 (2014)

13. N. Rajapaksha, A. Madanayake, L.T. Bruton, 2D space- time wave-digital multi-fan filter banks for signals consisting of multiple plane waves. Multidim. Syst. Sign. Process. 25(1), 17-39 (2014)

14. C. Guoying, W. Yan, Multi-mobile agent collaborative control data separation method in wireless sensor network. J. Comput. Appl. 35(4), 910-915 (2015)

15. L.I. Zuxiong, Periodic solution for a modified Leslie-Gower model with feedback control. Acta Mathematicae Applicatae Sinica 38(1), 37-52 (2015)

16. E.I. Elsedimy, M.Z. Rashad, M.G. Darwish, Multi-objective optimization approach for virtual machine placement based on particle swarm optimization in cloud data centers. J. Computat. Theor. Nanosci. 14(10), 5145-5150 (2017)

17. F. Abdessamia, T. Yu, Z.Z. Wei, et al., An improved particle swarm optimization for energy-efficiency virtual machine placement, international conference on cloud computing research and innovation. IEEE 17, 7-13 (2017)

18. B. Xu, Z. Peng, F. Xiao, et al., Dynamic deployment of virtual machines in cloud computing using multi-objective optimization. Soft. Comput. 19(8), 2265-2273 (2015)

19. D. Kumar, T. Mandal, Bi-objective virtual machine placement using hybrid of genetic algorithm and particle swarm optimization in cloud data center. Int. J. Appl. Eng. Res. 12(12), 12044-12051 (2017)

20. Bhardwaj S., Sahoo B. (2015). A particle swarm optimization approach for cost effective SaaS placement on cloud. International Conference on Computing, Communication \& Automation. IEEE, 15,686-690.

21. G. Karimi, H. Akbarpour, A. Sadeghzadeh, Multi objective particle swarm optimization based mixed size module placement in VLSI circuit design. Appl. Math. Inf. Sci. 9(3), 1485-1492 (2015)

22. D. Wang, X. Liu, Resource scheduling of cloud computing platform based on improved particle swarm optimization. Appl. Res. Comput. 8(1), 228-233 (2015)

23. J.R. Bezerra, G.C. Barroso, R.P.S. Leão, et al., Multiobjective optimization algorithm for switch placement in radial power distribution networks. IEEE Trans Power Deliver 30(2), 545-552 (2015)

24. W. Zhang, Y. Wang, Adaptive management and multi objective optimization of virtual machine placement in cloud computing. J. Comput. Theor. Nanosci. 3(12), 9683-9687 (2016)

25. Z. Huang, J. Tang, G. Shan, J. Ni, Y. Chen, C. Wang, An efficient passenger-hunting recommendation framework with multi-task deep learning. IEEE Internet Things J. (2019). https://doi.org/10.1109/JIOT.2019.2901759

26. Rui L,. Zheng Q., Li X., et al. (2017). A novel multi-objective optimization scheme for rebalancing virtual machine placement. IEEE, International Conference on Cloud Computing. IEEE, 17,710-717.

27. X. Wang, Y. Qi, Z. Wang, Design and implementation of SecPod: A framework for virtualization-based security systems. IEEE Trans Depend Secure Comput 16(1), 44-57 (2019)

28. Z. Chen, Y. Zhang, C. Wu, B. Ran, Understanding individualization driving states via latent Dirichlet allocation model. IEEE Intell Transportation Systems Magazine 11(2), 41-53 (2019) 
29. P. Zheng, Q. Yong, Z. Yangfan, C. Pengfei, An automatic framework for detecting and characterizing performance degradation of software systems. IEEE Trans Reliability 63(4), 927-943 (2014)

30. J. Han, W. Zang, L. Liu, et al., Risk-aware multi-objective optimized virtual machine placement in cloud. J. Comput. Secur. $18(5), 1-24(2018)$

31. Z. Huang, X. Xu, J. Ni, H. Zhu, C. Wang, Multimodal representation learning for recommendation in internet of things. IEEE Internet Things J. 6(6), 10675-10685 (2019)

32. T. Zhou, J. Zhang, Analysis of commercial truck drivers' potentially dangerous driving behaviors based on 11-month digital tachograph data and multilevel modeling approach. Accid. Anal. Prev. 132(1), 105256 (2019)

33. B. Wu, T.L. Yip, X. Yan, C. Guedes Soares, Fuzzy logic based approach for ship-bridge collision alert system. Ocean Eng. 187(1), 106152 (2019)

\section{Publisher's Note}

Springer Nature remains neutral with regard to jurisdictional claims in published maps and institutional affiliations.

Submit your manuscript to a SpringerOpen ${ }^{\circ}$ journal and benefit from:

- Convenient online submission

- Rigorous peer review

- Open access: articles freely available online

- High visibility within the field

- Retaining the copyright to your article

Submit your next manuscript at $\boldsymbol{\nabla}$ springeropen.com 